\title{
(C) OPEN ACCESS \\ Evaluation of iris and iridociliary body lesions with anterior segment optical coherence tomography versus ultrasound B-scan
}

\author{
Scott C Hau, ${ }^{1}$ Vasilios Papastefanou, ${ }^{1,2}$ Shima Shah, ${ }^{1}$ Mandeep S Sagoo, ${ }^{1,2,3}$ \\ Marie Restori, ${ }^{1}$ Victoria Cohen ${ }^{1,2}$
}

\begin{abstract}
${ }^{1}$ NIHR Biomedical Research Centre in Ophthalmology Moorfields Eye Hospital NHS Foundation Trust, London, UK ${ }^{2}$ Ocular Oncology Service, St. Bartholomew's Hospital, London, UK

${ }^{3} \mathrm{UCL}$ Institute of Ophthalmology, London, UK
\end{abstract}

\section{Correspondence to} Scott C Hau, Moorfields Eye Hospital NHS Foundation Trust 162 City Road, London EC1V 2PD, UK;

scott.hau@moorfields.nhs.uk

Received 9 March 2014 Revised 23 May 2014 Accepted 13 July 2014 Published Online First 4 August 2014
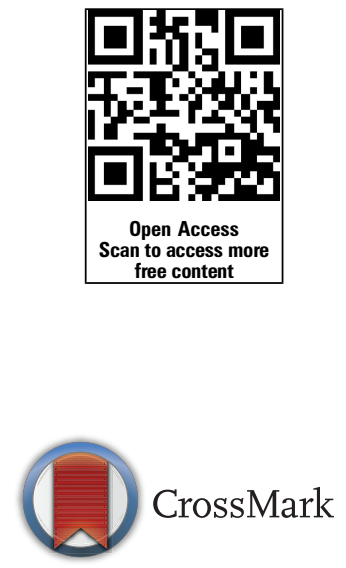

To cite: Hau SC, Papastefanou V, Shah S, et al. Br J Ophthalmol 2015;99:81-86.

\section{ABSTRACT}

Aims To compare anterior segment optical coherence tomography (AS-OCT) with ultrasound B-scan (USB) in evaluating iris and iridociliary body lesions.

Methods Image features and resolution comparison between AS-OCT and USB in 126 patients (126 eyes) presenting with iris or iridociliary body lesion.

Bland-Altman plots were generated to assess the level of agreement between the two techniques.

Results The three most common diagnoses were iris naevi (62 (49.2\%)), iris pigment epithelial cysts (23 $(18.3 \%))$ and iris melanoma (11 (8.7\%)). Image feature comparison for USB was better than AS-OCT in visualising all tumour margins (81 (64.3\%) vs 59 $(46.8 \%))$, posterior tumour margin (54 (42.9\%) vs 16 $(12.7 \%))$ and producing less posterior shadowing (121 (96\%) vs $43(34.1 \%))$. Image resolution comparison revealed USB to be slightly better for resolving the overall tumour (45 (35.7\%) vs $43(34.1 \%))$ and posterior tumour surface $(70(55.6 \%)$ vs 32 (25.4\%)) but AS-OCT was better for resolving the anterior $(62(49.2 \%)$ vs $4(3.2 \%))$ and lateral tumour surface $(62(49.2 \%)$ vs $31(24.6 \%))$. Comparing the three most common diagnoses, USB was better for visualising iris pigment epithelial cysts $(12(52.2 \%)$ vs 2 $(8.7 \%))$ and iris melanoma $(7(63.6 \%)$ vs $1(9.1 \%))$ but AS-OCT was better $(28(45.2 \%)$ vs $15(24.2 \%))$ for visualising iris naevi. Bland-Altman plots showed good agreement between the two techniques for lesions smaller than $3 \mathrm{~mm}$ in base and $2 \mathrm{~mm}$ in elevation. Conclusions AS-OCT is superior to USB for imaging small lesions pertaining to the anterior iris but USB is better for imaging larger iris lesions with posterior or ciliary body extension.

\section{INTRODUCTION}

Anterior segment optical coherence tomography (AS-OCT) is a relatively new imaging modality that allows detailed assessment of the anterior chamber structures by obtaining rapid high-resolution crosssectional images without direct contact with the eye. ${ }^{1}$ The technology is based on low-coherence interferometry, and it measures the time delay and intensity of light back-reflected from tissue structures at various depths compared with a reference standard. Image creation with OCT imaging is analogous to ultrasound B-scan (USB), whereby rapid successive axial measurements (A-scans) are recorded at different transverse locations. The axial measurements indicate the echo time delay of backreflected and back-scattered light from structures within the tissue and they are then compiled together to form a two-dimensional cross-sectional image. $^{2}$

USB is a well-established imaging modality for quantitative assessment of ocular structures in the globe. However, the spatial resolution of ultrasound systems is limited because of the low frequency $(10-20 \mathrm{MHz})$ in which they operate. High-frequency ultrasound biomicroscopy (UBM), which operates in the range of $30-50 \mathrm{MHz}$, can achieve higher resolution than conventional ultrasound but with poorer tissue penetration. Therefore, the clinical use of UBM is limited to examining the anterior segment of the eye. Compared with USB, the axial resolution of timedomain AS-OCT is higher $(18 \mu \mathrm{m}$ in Visante vs $25 \mu \mathrm{m}$ in $50 \mathrm{MHz}$ UBM). ${ }^{3}$ More recently, with the development of spectral/Fourier-domain OCT, the axial resolution with some AS-OCT devices is as high as $5-7 \mu \mathrm{m} .^{12}$

Although several studies have been published comparing UBM with AS-OCT in evaluating anterior segment tumours, ${ }^{4-7}$ there is little in the literature comparing USB with AS-OCT in the evaluation of iris and iridociliary body lesions. This is an important consideration as USB and OCT devices are often more widely available than UBM in general ophthalmic units. The aim of this study is to evaluate which imaging modality (AS-OCT vs USB) provides better visualisation and characterisation of a large cohort of iris and iridociliary body lesions.

\section{METHODS}

This study was approved by the Research \& Ethics Committee of Moorfields Eye Hospital, UK, and it adhered to the tenets of Declaration of Helsinki.

A retrospective case note review was carried out of all patients with iris or iridociliary body lesions imaged with both AS-OCT and USB who presented to the ocular oncology service at Moorfields Eye Hospital between the periods of October 2009 to September 2011. The inclusion criteria were patients who had either an iris or iridociliary body lesion. Patients with other anterior or posterior segment lesions or tumours were excluded. The diagnosis of the lesions was made clinically based on a combination of clinical examination, gonioscopy, transillumination, indirect ophthalmoscopic examination with scleral depression and imaging findings. Melanomas were defined as lesions $>3 \mathrm{~mm}$ in diameter and $1 \mathrm{~mm}$ in thickness, iris stroma and ciliary body invasion, and if three of the following five features were present-documented growth, secondary 
glaucoma, secondary cataract, prominent vascularity and ectropion uvea. $^{8}$

\section{Imaging}

Imaging was performed with both USB and AS-OCT on the first visit in all the cases. The USBs were performed by a consultant medical physicist (MR) using the Acuson Sequoia 512 (Siemens AG, Munich, Germany) with a $25 \mathrm{~mm}$ footprint $14 \mathrm{MHz}$ linear B-scan array probe. The system has a depth of penetration to the orbital wall, and it has a facility to expand or zoom into a region of interest in the anterior chamber within the whole $\mathrm{B}$-scan range of the eye and orbit. The probe was smeared with a coupling gel (Aquasonic 100, Parker Lab, New Jersey, USA) and placed on the patient's closed eyelids directly anterior to the lesion. All scans were performed with the lids closed to enhance patient comfort. The gain setting was adjusted to maximise the quality of the image, and it varied from -20 to $+20 \mathrm{~dB}$ depending on the thickness of the eyelid. The images were taken in both the longitudinal (radial) and transverse directions, and care was taken to ensure the full extent of the lesions was seen before image recording. One good quality image from each scanning direction was used for analysis. The principles of AS-OCT have been described in detail elsewhere. ${ }^{9}$ AS-OCT images were performed by one experienced operator (SS) using the Visante (Carl Zeiss Meditec, Dublin, California, USA) timedomain OCT system. All the images were obtained in both the longitudinal (radial) and transverse directions. The AS-OCT images were obtained using the anterior segment single (ASS) acquisition mode and anterior segment high resolution (ASHR) mode. If it was not possible to obtain an image using the ASHR mode then only images obtained with the ASS mode were used for analysis. To minimise image distortion and measurement error, care was taken to ensure the OCT light source was perpendicular to the iris or iridociliary lesion and that a bright vertical flare line crossing the corneal vertex was seen. One good quality image was selected from each case and analysed.

\section{Image comparison}

The USB and AS-OCT images were analysed separately by two independent observers (VP and SCH), respectively. The following image features were compared between the two imaging modalities and recorded: acoustic/reflectivity features (hollow, solid, undetectable), internal tumour pattern (heterogeneous, homogeneous), visibility of tumour surface (anterior, medial, lateral and posterior), were all margins seen, degree of posterior shadowing, presence of pigmentation, size of the lesions and image resolution. Resolution was defined as the amount of detail and definition that could be resolved in each image with the two imaging modalities. Image resolution comparison included overall tumour visualisation, internal tumour structures, tumour surface (anterior, posterior, medial and lateral), tumour colour (pigmented or non-pigmented) and location of lesion (iris, ciliary body or iridociliary). For qualitative assessment of the lesions, the images were graded as good, fair, poor or undetectable by each observer. The grading of the images was then cross-tabulated and compared between the two imaging modalities. If both observers gave the same grading for a particular case, then this was classified as 'no difference' between the two images. Bland-Altman plots were used to assess the level of agreement in the size of the base and elevation of the lesions between the two imaging modalities. ${ }^{10}$ Finally, a comparison of image resolution for the three most common diagnoses was also conducted. Patient demographics including age and sex and clinical diagnosis were also recorded.

\section{RESULTS}

A total of 126 consecutive patients (126 eyes) were included in the study. The mean age of the patient group was 57.8 (SD \pm 18.7 ; range 20-92); 56 (44.4\%) were men and $70(55.6 \%)$ women. The list of diagnoses detectable by USB and AS-OCT is shown in table 1 .

In total, AS-OCT was able to detect more lesions than USB (103 (81.7\%) vs $90(71.4 \%))$, especially in imaging iris lesions, but it was unable to detect any of the ciliary body lesions. USB was unable to image iris freckles, iris nodules, peripheral anterior synechiae and angle naevi. The frequency of the lesion configurations is as follows: $63(50 \%)$ dome shape, 32 flat $(25.4 \%)$, 13 mushroom (10.4\%) shape, 9 derby hat $(7.1 \%)$ and $9(7.1 \%)$ spherical in shape. The most common tumour location was in the inferior temporal quadrant of the iris $(34(27 \%))$.

The imaging features for USB and AS-OCT are given in table 2.

USB was superior in terms of the visibility of all tumour margins $(64.3 \%$ vs $46.8 \%)$ but AS-OCT fared better in visualising the anterior tumour margin with more images being graded 'good' compared with USB ( $88.9 \%$ vs $50 \%)$. USB was better in visualising the posterior tumour margin $(42.9 \%$ vs $12.7 \%)$ and also produced much less posterior shadowing compared with AS-OCT (96\% vs 34.1\%). The mean elevation of the lesions was higher with USB but the mean longitudinal base was lower compared with AS-OCT.

A comparison for better image resolution between the two techniques is shown in table 3 .

USB offered slightly better overall tumour resolution $(35.7 \%$ vs $34.1 \%$ ) but it was less able to resolve the internal structures $(30.2 \%$ vs $43.7 \%)$. The image resolution of the anterior surface (49.2\% vs $3.2 \%)$ and lateral surface $(49.2 \%$ vs $24.6 \%)$ was superior with AS-OCT, whereas USB was significantly better in resolving the posterior tumour surface $(55.6 \%$ vs $25.4 \%)$. In addition, USB was better at resolving the lesions irrespective of the degree of pigmentation.

Table 1 Diagnoses detectable by ultrasound B-scan and anterior segment optical coherence tomography

\begin{tabular}{lccl}
\hline Diagnosis & $\begin{array}{l}\text { Number (\%) } \\
\mathrm{N}=126\end{array}$ & $\begin{array}{l}\text { Detectable by } \\
\text { USB number (\%) }\end{array}$ & $\begin{array}{l}\text { Detectable by } \\
\text { AS-OCT number (\%) }\end{array}$ \\
\hline Angle naevus & $2(1.6)$ & 0 & $1(50)$ \\
Ciliary body adenoma & $2(1.6)$ & $2(100)$ & 0 \\
Ciliary body melanoma & $1(0.8)$ & $1(100)$ & 0 \\
Ciliary body naevus & $1(0.8)$ & $1(100)$ & 0 \\
Iris amelanotic naevus & $3(2.4)$ & $2(67)$ & $3(100)$ \\
Iris freckle & $2(1.6)$ & 0 & $2(100)$ \\
Iris haemangioma & $3(2.4)$ & $1(33)$ & $2(67)$ \\
Iris leiomyoma & $1(0.8)$ & $1(100)$ & $1(100)$ \\
Iris melanocytoma & $3(2.4)$ & $3(100)$ & $3(100)$ \\
Iris melanoma & $11(8.7)$ & $11(100)$ & $11(100)$ \\
Iris naevus & $62(49.2)$ & $43(69.4)$ & $54(87.1)$ \\
Iris nodules & $1(0.8)$ & 0 & $1(100)$ \\
IPE & $23(18.3)$ & $21(91.3)$ & $17(73.9)$ \\
Iris plasmacytoma & $1(0.8)$ & $1(100)$ & $1(100)$ \\
Iris ring melanoma & $2(1.6)$ & $1(50)$ & $1(50)$ \\
Iris stromal cyst & $1(0.8)$ & $1(50)$ & $1(50)$ \\
No definite diagnosis & $4(3.2)$ & $1(25)$ & $2(50)$ \\
PAS & $3(2.4)$ & 0 & $3(100)$ \\
\hline AS-OCT, anterior segment optical coherence tomography; IPE, iris pigment epithelial \\
Cyst; PAS, peripheral anterior synechiae; USB, ultrasound B-scan.
\end{tabular}


Table 2 Ultrasound B-scan versus anterior segment optical coherence tomography: image features

\begin{tabular}{|c|c|c|c|c|c|c|}
\hline Features & $\begin{array}{l}\text { Ultrasound B scan } \\
\mathrm{N}=126 \\
\text { Number (\%) }\end{array}$ & & & $\begin{array}{l}\text { AS-OCT } \\
\mathrm{N}=126 \\
\text { Number (\%) }\end{array}$ & & \\
\hline \multicolumn{7}{|l|}{ Acoustic features } \\
\hline Hollow & $38(30.2)$ & & & $30(23.8)$ & & \\
\hline Solid & $51(40.5)$ & & & $86(68.3)$ & & \\
\hline Lesion undetectable & 37 (29.4) & & & $10(7.9)$ & & \\
\hline \multicolumn{7}{|l|}{ Internal pattern } \\
\hline Heterogeneous & $39(31)$ & & & $29(23.1)$ & & \\
\hline Homogeneous & $50(39.7)$ & & & $87(69)$ & & \\
\hline Lesion undetectable & $37(29.4)$ & & & $10(7.9)$ & & \\
\hline Visibility & Good & Fair & Undetectable & Good & Fair & Undetectable \\
\hline Anterior & $63(50)$ & $25(19.8)$ & $38(30.2)$ & $112(88.9)$ & $10(7.9)$ & $4(3.2)$ \\
\hline Medial & $57(45.2)$ & $29(23)$ & 40 (31.7) & $59(46.8)$ & $44(34.9)$ & $23(18.3)$ \\
\hline Lateral & $51(40.5)$ & $34(27)$ & $41(32.5)$ & $60(47.6)$ & $43(34.1)$ & $23(18.3)$ \\
\hline Posterior & $54(42.9)$ & $33(26.2)$ & $39(31)$ & $16(12.7)$ & $57(45.2)$ & $53(42.1)$ \\
\hline \multicolumn{7}{|l|}{ All margins seen } \\
\hline Yes & $81(64.3)$ & & & $59(46.8)$ & & \\
\hline No & $45(35.7)$ & & & $67(53.2)$ & & \\
\hline \multicolumn{7}{|l|}{ Posterior shadowing } \\
\hline None & $121(96)$ & & & $43(34.1)$ & & \\
\hline Yes-trace & $2(1.6)$ & & & $12(9.5)$ & & \\
\hline Yes-mild & $1(0.8)$ & & & $22(17.5)$ & & \\
\hline Yes-moderate & 0 & & & $22(17.5)$ & & \\
\hline Yes-severe & $2(1.6)$ & & & 27 (21.4) & & \\
\hline \multicolumn{7}{|l|}{ Elevation (mm) } \\
\hline Mean (SD, range) & $1.8( \pm 1.4,0.3$ to 5.8$)$ & & & $1.0( \pm 1.1,0$. & & \\
\hline \multicolumn{7}{|l|}{ Longitudinal base (mm) } \\
\hline Mean (SD, range) & $2.6( \pm 1.5,1.0$ to 7.8$)$ & & & $2.8( \pm 1.6,0$. & & \\
\hline \multicolumn{7}{|l|}{ Transverse base $(\mathrm{mm})$} \\
\hline Mean (SD, range) & $2.9( \pm 0.8,0.8$ to 12.8$)$ & & & $2.9( \pm 1.6,0$ & & \\
\hline
\end{tabular}

AS-OCT, anterior segment optical coherence tomography.

A comparison of the three most common diagnoses is shown in table 4.

Overall, USB was better for visualising iris pigment epithelial (IPE) cysts $(52.2 \%$ vs $8.7 \%)$ and iris melanoma $(63.6 \%$ vs $9.1 \%)$ but less so for iris naevi $(24.2 \%$ vs $45.2 \%)$. USB was also superior for resolving the posterior surface and produced less posterior shadowing than AS-OCT in all three conditions. In

Table 3 Ultrasound B-scan versus anterior segment optical coherence tomography: image resolution comparison

\begin{tabular}{|c|c|c|c|}
\hline $\begin{array}{l}\text { Categories } \\
N=126\end{array}$ & $\begin{array}{l}\text { Better with } \\
\text { USB number } \\
\text { (\%) }\end{array}$ & $\begin{array}{l}\text { Better with } \\
\text { AS-OCT number } \\
\text { (\%) }\end{array}$ & $\begin{array}{l}\text { No difference } \\
\text { number (\%) }\end{array}$ \\
\hline $\begin{array}{l}\text { Overall tumour } \\
\text { visualisation }\end{array}$ & 45 (35.7) & $43(34.1)$ & $38(30.2)$ \\
\hline Anterior surface & $4(3.2)$ & $62(49.2)$ & 60 (47.6) \\
\hline Posterior surface & $70(55.6)$ & $32(25.4)$ & $24(19)$ \\
\hline Lateral surface & $31(24.6)$ & $62(49.2)$ & $33(26.2)$ \\
\hline Internal structures & $38(30.2)$ & $55(43.7)$ & $33(26.2)$ \\
\hline $\begin{array}{l}\text { Pigmented lesion } \\
(\mathrm{N}=85)\end{array}$ & 32 (37.6) & $26(30.6)$ & $27(31.8)$ \\
\hline $\begin{array}{l}\text { Non-pigmented } \\
\text { lesion }(\mathrm{N}=41)\end{array}$ & $19(46.4)$ & $11(26.8)$ & $11(26.8)$ \\
\hline Location of lesion & $46(36.5)$ & $38(30.2)$ & $42(33.3)$ \\
\hline
\end{tabular}

AS-OCT, anterior segment optical coherence tomography; USB, ultrasound B-scan. terms of lesion size, both mean elevation and base were larger with USB for measuring IPE cysts and iris melanoma, whereas for iris naevus, the mean base measured greater with AS-OCT. The 19 (30.6\%) iris naevi that were not detected by USB had base values $\leq 2 \mathrm{~mm}$ and elevation $\leq 0.6 \mathrm{~mm}$. The two IPE cysts that were not detected by USB but were imaged with AS-OCT had elevation values of 0.8 and $1.0 \mathrm{~mm}$, respectively.

Bland-Altman plots for base and elevation measurements are shown in figure 1 . The base measurements obtained with USB were on average lower but the elevation measurements were higher compared with AS-OCT. For base measurements, the agreement was better when the values were $<3 \mathrm{~mm}$, whereas for elevation values, the agreement was better when it was $<2 \mathrm{~mm}$. Above these values, there was an increase in dispersion indicating worsening agreement between the two techniques. A composite image of AS-OCT, anterior segment photograph, USB images for iris melanoma, iris naevus and IPE cyst is shown in figure 2 .

\section{DISCUSSION}

Anterior segment imaging is an important diagnostic tool in the management of patients with iris and iridociliary body lesions. Imaging is of major importance in distinguishing between tumours and pseudotumours. This allows the characterisation of these lesions, often avoiding intraocular biopsy, and also permits serial evaluations. This becomes important in treated tumours, where a response can be monitored, and in suspicious lesions, 


\section{Clinical science}

Table 4 Ultrasound B-scan versus anterior segment optical coherence tomography: image comparison for the three most common diagnoses

\begin{tabular}{|c|c|c|c|c|c|c|c|c|c|}
\hline \multirow[b]{2}{*}{ Categories } & \multicolumn{3}{|c|}{$\begin{array}{l}\text { Iris naevus } \\
\mathrm{N}=62^{*} \\
\text { Number (\%) }\end{array}$} & \multicolumn{3}{|c|}{$\begin{array}{l}\text { IPE cyst } \\
\mathrm{N}=23 \\
\text { Number (\%) }\end{array}$} & \multicolumn{3}{|c|}{$\begin{array}{l}\text { Iris melanoma } \\
\mathrm{N}=11^{\dagger} \\
\text { Number (\%) }\end{array}$} \\
\hline & USB & AS-OCT & ND & USB & AS-OCT & ND & USB & AS-OCT & ND \\
\hline Better overall tumour visualisation & $15(24.2)$ & $28(45.2)$ & $19(30.6)$ & $12(52.2)$ & $2(8.7)$ & $9(39.1)$ & $7(63.6)$ & $1(9.1)$ & $3(27.3)$ \\
\hline Better anterior surface resolution & 0 & $37(59.7)$ & $25(40.3)$ & $1(4.3)$ & $7(30.5)$ & $15(65.2)$ & 0 & $1(9.1)$ & $10(90.9)$ \\
\hline Better posterior surface resolution & $30(48.4)$ & 19 (30.6) & $13(21)$ & $15(65.2)$ & $5(21.7)$ & $3(13.1)$ & $9(81.8)$ & $1(9.1)$ & $1(9.1)$ \\
\hline Better lateral surface resolution & $6(9.7)$ & $44(71)$ & $12(19.4)$ & $14(60.9)$ & $4(17.4)$ & $5(21.7)$ & $3(27.3)$ & $3(27.3)$ & $5(45.4)$ \\
\hline Better internal structure resolution & $8(12.9)$ & $37(59.7)$ & $17(27.4)$ & $14(60.9)$ & $3(13)$ & $6(26.1)$ & $6(54.5)$ & $2(18.2)$ & $3(27.3)$ \\
\hline Better resolution if pigmented & $11(19.6)$ & $27(48.2)$ & $18(32.1)$ & NA & NA & NA & $6(66.7)$ & $2(22.2)$ & $1(11.1)$ \\
\hline Better resolution if non-pigmented & $4(66.6)$ & $1(16.7)$ & $1(16.7)$ & NA & NA & NA & 0 & 0 & $2(100)$ \\
\hline Less posterior shadowing & $43(69.4)$ & $1(1.6)$ & $18(29)$ & $6(26.1)$ & $2(8.7)$ & $15(65.2)$ & $10(90.9)$ & 0 & $1(9.1)$ \\
\hline $\begin{array}{l}\text { Elevation (mm) } \\
\text { Mean (SD) }\end{array}$ & $0.8( \pm 0.9)$ & $0.6( \pm 0.7)$ & NA & $1.7( \pm 1.6)$ & $0.9( \pm 1.5)$ & NA & $2.6( \pm 1.5)$ & $0.8( \pm 10.8)$ & NA \\
\hline $\begin{array}{l}\text { Longitudinal base }(\mathrm{mm}) \\
\text { Mean (SD) }\end{array}$ & $1.3( \pm 1.2)$ & $2.2( \pm 1.6)$ & NA & $2.2( \pm 1.9)$ & $1.9( \pm 1.9)$ & NA & $3.6( \pm 1.3)$ & $3.0( \pm 2.2)$ & NA \\
\hline $\begin{array}{l}\text { Transverse base (mm) } \\
\text { Mean (SD) }\end{array}$ & $1.4( \pm 1.3)$ & $2.1( \pm 1.5)$ & NA & $2.3( \pm 1.9)$ & $2.0( \pm 2.2)$ & NA & $3.7( \pm 1.4)$ & $3.3( \pm 1.6)$ & NA \\
\hline
\end{tabular}
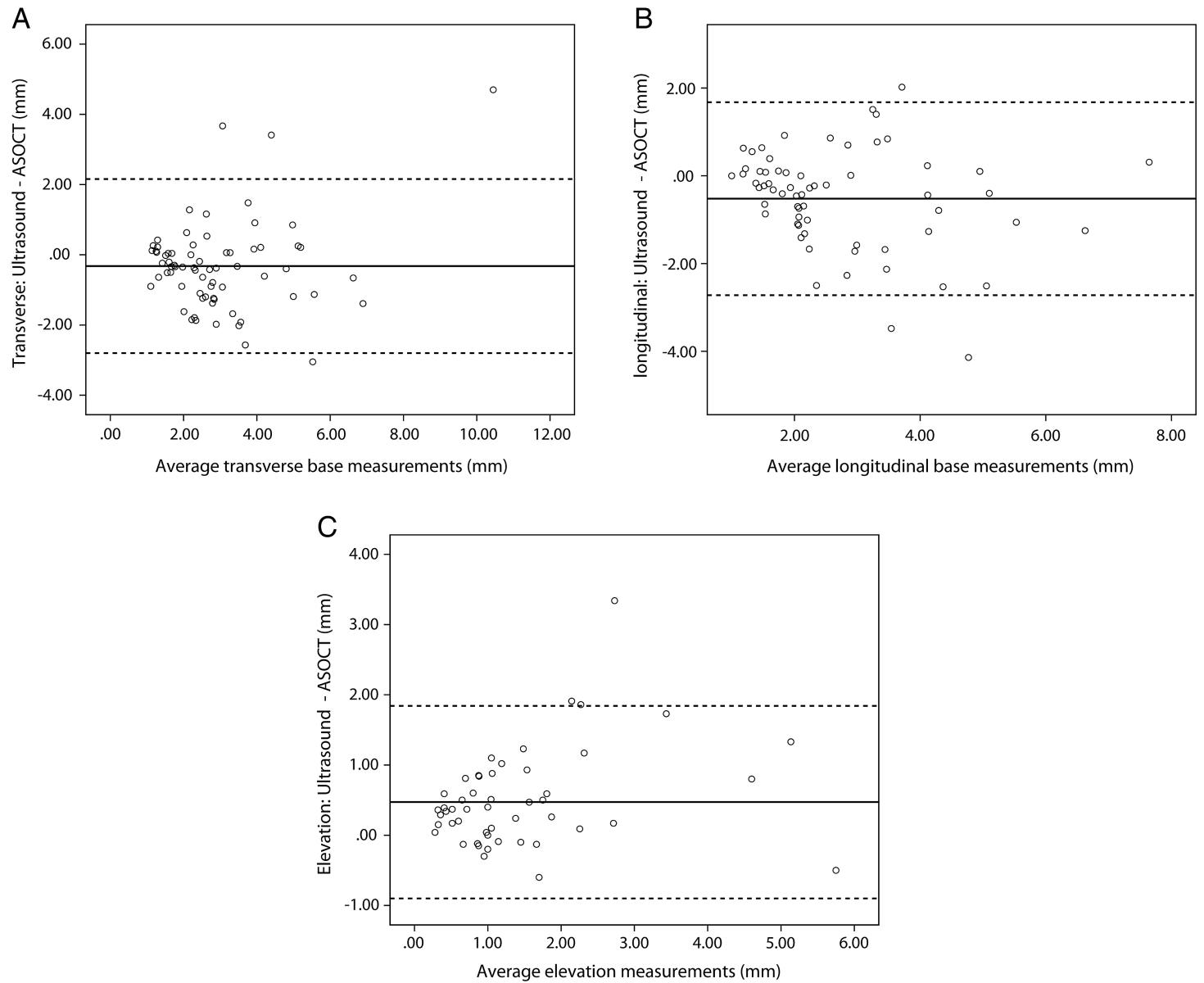

Figure 1 (A) Bland-Altman plot for the transverse base measurements showing the difference between ultrasound and anterior segment optical coherence tomography (AS-OCT) against the average measurements for the two imaging modalities. The solid line is the mean difference $(-0.32 \mathrm{~mm})$. The broken lines indicate the $95 \%$ limits of agreement. (B) Bland-Altman plot for the longitudinal (radial) base measurements showing the difference between ultrasound and AS-OCT against the average measurements for the two imaging modalities. The solid line is the mean difference $(-0.52 \mathrm{~mm})$. The broken lines indicate the $95 \%$ limits of agreement. (C) Bland-Altman plot for the elevation measurements showing the difference between ultrasound and AS-OCT against the average measurements for the two imaging modalities. The solid line is the mean difference $(0.47 \mathrm{~mm})$. The broken lines indicate the $95 \%$ limits of agreement. 


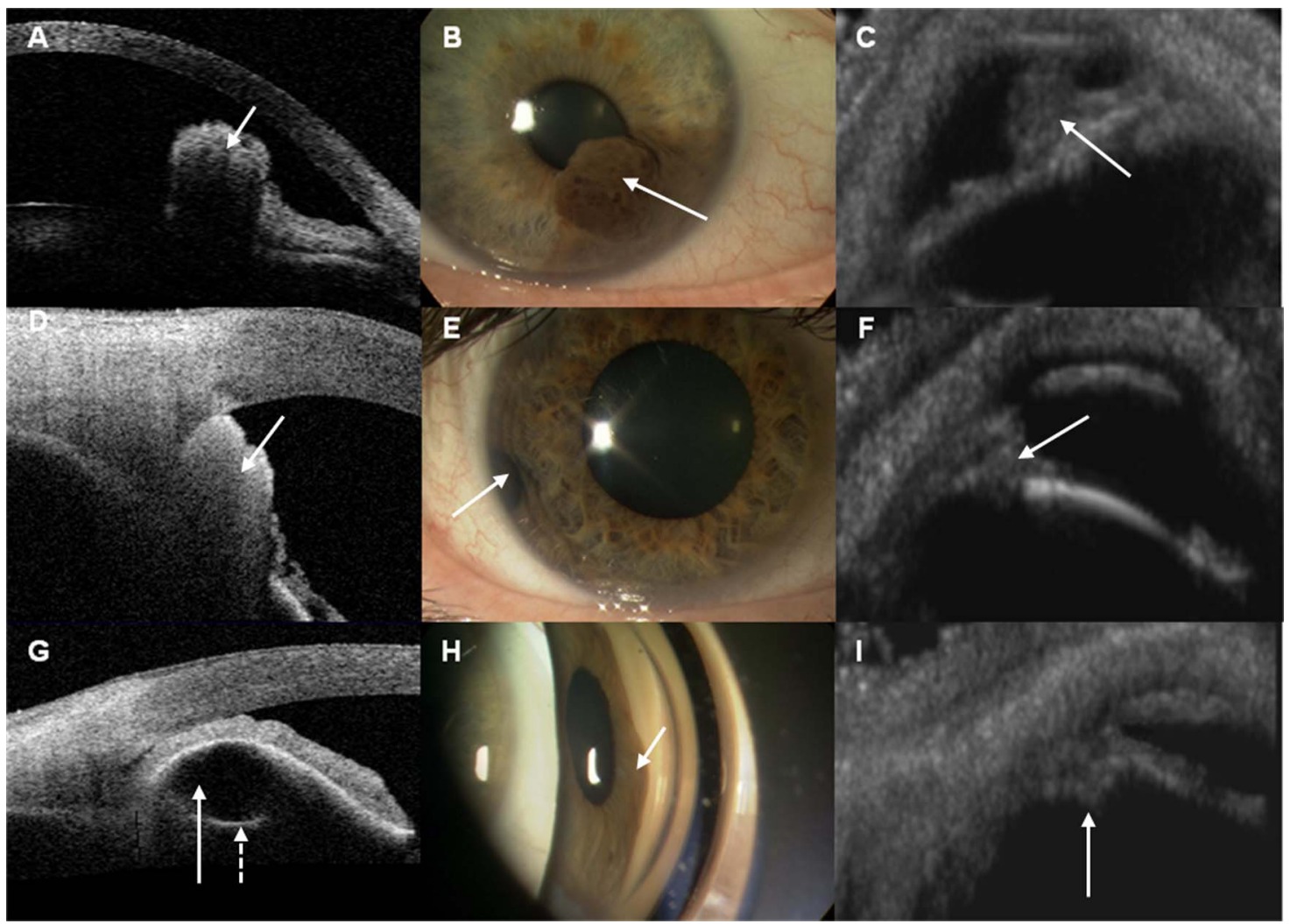

Figure 2 Composite image of anterior segment optical coherence tomography (AS-OCT), anterior segment photograph and ultrasound B-scan (USB) for three different diagnoses. Images A-C (arrows) show an iris melanoma (B) with anterior visualisation with AS-OCT (A) and complete visualisation with USB (C). Images D-F (arrows) show an iris naevus (E) with anterior visualisation using AS-OCT (D) and complete visualisation of the lesion with USB (F). Images G-I show a gonioscopic view of an iris pigment epithelial cyst (H) showing the central hyporeflective region (arrow) and a surrounding curved hyperreflective line (broken arrow) with near complete visualisation of the lesion with AS-OCT (G) but not clearly detectable with USB (I).

where precise documentation of growth is used as an important indicator of malignant transformation. ${ }^{11-13}$ It is of even more importance when a newer modality becomes available to compare the features with more established techniques. This helps to define the precise role of the available technologies, whether they are interchangeable or complimentary.

USB is a well-established imaging modality for measuring and quantifying a range of intraocular lesions and tumours in the eye. ${ }^{14-18}$ The role of UBM has also been studied. Marigo et al found that there was good correlation between UBM and histopathological findings in terms of reflectivity, size and degree of tumour extension. ${ }^{19}$ Conway et al compared USB with UBM and found that ciliary body involvement was identified more frequently in iris and choroidal melanoma using UBM. ${ }^{13}$ However, USB was still recommended for large iris melanomas, to detect very large posterior extension or to characterise internal acoustic pattern. ${ }^{13}$ Hence, UBM has not replaced USB, but is a useful adjunct.

There have been some reports in the literature comparing AS-OCT with UBM in imaging anterior segment lesions. Pavlin et al found AS-OCT was only useful in small hypopigmented tumours confined to the iris, whereas UBM is better for highly pigmented and ciliary body tumours. ${ }^{5}$ Razzaq compared AS-OCT, slit-lamp OCT (SL-OCT, Heidelberg engineering, Germany), Pentacam (Oculus, Germany) and UBM in imaging melanocytic iris tumours. They reported that AS-OCT images were superior to SL-OCT and Pentacam, and the images were comparable with those obtained with UBM unless there was ciliary body extension, where UBM was superior. ${ }^{20}$ Siahmed et al reported a series of 61 iris tumours imaged with both AS-OCT and UBM, advocating the latter for measuring growth progression. ${ }^{6}$ Bianciotto et al compared 200 eyes with AS-OCT and UBM, with UBM offering better visualisation of the posterior margin and better images of the entire tumour configuration. ${ }^{4}$

In the present study, we have compared the readily available techniques of USB and AS-OCT. Our findings indicate that conventional USB is superior in visualising the entire tumour and posterior tumour margin with minimal posterior shadowing, similar to studies comparing UBM with AS-OCT. ${ }^{4}$ We found AS-OCT superior at imaging the anterior and lateral surface of iris lesions. This is by virtue of the fact that the time-domain AS-OCT has a higher axial resolution (18 microns) than USB and, therefore, was better at imaging small anterior iris surface lesions such as naevi. Our findings suggest that AS-OCT is better than USB for imaging iris naevus measuring $\leq 2 \mathrm{~mm}$ in base and $0.6 \mathrm{~mm}$ in elevation. However, for larger lesions such as pigmented iris melanoma or lesions with posterior extension, USB was superior in visualising the whole tumour configuration and internal structures because of the better penetration of sound waves versus light energy in heavily pigmented lesions. The restriction of light penetration results in posterior shadowing and difficulties in delineating the posterior edge of the lesion. We found this to be the main factor in the lower AS-OCT elevation data in the iris melanoma group. Further limitation with AS-OCT includes measurement error with off-axis imaging and the use of specialised dewarping algorithm 
based on the refractive index of the cornea to obtain measurement values. ${ }^{2}$ Therefore, caution must be exercised when the measurements are compared with those obtained with other AS-OCT and ultrasound devices.

The transverse base measurements were comparable between the two instruments, but we found the longitudinal base values were smaller and the elevation greater with USB; the better visualisation of the complete lesion with ultrasound may explain the larger values. Based on this, lesions larger than $3 \mathrm{~mm}$ in elevation maybe better imaged and followed with USB than AS-OCT. However, as the USB in this study was performed with closed eyelids, it is possible the measurement of the lesions was taken in a slightly different position compared with AS-OCT. The role of AS-OCT points more towards an adjunctive imaging modality to USB, rather than one that replaces it.

Accurate location and delineation of tumour margins are essential for monitoring growth in benign lesions or in planning treatment for neoplastic lesions. In agreement with previous studies using UBM, USB is superior to AS-OCT in imaging ciliary body lesions. ${ }^{4-6} 13$ However, due to the lower frequency sound waves USB operates, the resolution and the ability in detecting small iris lesions are inferior compared with UBM. This is evident by the fact that nearly $30 \%$ of iris lesions were not detectable with USB in this study (table 1). Furthermore, we used a $14 \mathrm{MHz}$ probe for imaging the lesions, which is higher than the more conventional $10 \mathrm{MHz}$ probes. The ability to detect small iris lesions may even be poorer with the lower frequency probes.

Although UBM has some distinct advantages over USB and AS-OCT, issues such as instrument availability, time constraints and the expertise required in using this imaging modality may limit its use in some institutions. ${ }^{13}$ USB is available in most ocular oncology units, and it is still the imaging of choice when the lesion is large with evidence of posterior extension and where detailed characterisation of the acoustic pattern of the lesion is required. ${ }^{13}$

In conclusion, AS-OCT is superior to USB for imaging small iris lesions pertaining to the anterior iris but USB is better for imaging larger iris lesions with posterior or ciliary body extension.

Acknowledgement The authors would like to thank Dr Catey Bunce for her statistical advice.

Contributors We would like to confirm that all authors provided (1) substantial contributions to the conception or design of the work, the acquisition of data and the interpretation of data; (2) drafting of the manuscript and revising it critically for important intellectual content; (3) final approval of the version to be published; and (4) an agreement to be accountable for all aspects of the work in ensuring that questions related to the accuracy or integrity of any part of the work are appropriately investigated and resolved.

Competing interests None.

Ethics approval Research \& Ethics Committee of Moorfields Eye Hospital.

Provenance and peer review Not commissioned; externally peer reviewed.
Open Access This is an Open Access article distributed in accordance with the Creative Commons Attribution Non Commercial (CC BY-NC 4.0) license, which permits others to distribute, remix, adapt, build upon this work non-commercially, and license their derivative works on different terms, provided the original work is properly cited and the use is non-commercial. See: http://creativecommons.org/ licenses/by-nc/4.0/

\section{REFERENCES}

1 Ramos JL, Li Y, Huang D. Clinical and research applications of anterior segment optical coherence tomography - a review. Clin Experiment Ophthalmol 2009;37:81-9.

2 Huang D, Duker JS, Fujimoto JG, et al. Imaging the eye from front to back with RTVue Fourier Domain optical coherence tomography. Thorofare, NJ: Slack Incorporate, 2010

3 Ishikawa H. Anterior segment imaging for glaucoma: OCT or UBM? Br J Ophthalmol 2007;91:1420-1.

4 Bianciotto C, Shields CL, Guzman JM, et al. Assessment of anterior segment tumors with ultrasound biomicroscopy versus anterior segment optical coherence tomography in 200 cases. Ophthalmology 2011;118:1297-302.

5 Pavlin CJ, Vásquez LM, Lee R, et al. Anterior segment optical coherence tomography and ultrasound biomicroscopy in the imaging of anterior segment tumors. Am J Ophthalmol 2009;147:214-19.

6 Siahmed K, Berges O, Desjardins L, et al. [Anterior segment tumor imaging: advantages of ultrasound $(10,20$ and $50 \mathrm{MHz})$ and optical coherence tomography]. J Fr Ophtalmol 2004;27:169-73.

7 Krema H, Santiago RA, Gonzalez JE, et al. Spectral-domain optical coherence tomography versus ultrasound biomicroscopy for imaging of nonpigmented iris tumors. Am J Ophthalmol 2013;156:806-12.

8 Shields JA, Sanborn GE, Augsburger JJ. The differential diagnosis of malignant melanoma of the iris. A clinical study of 200 patients. Ophthalmology 1983;90:716-20.

9 Radhakrishnan S, Rollins AM, Roth JE, et al. Real-time optical coherence tomography of the anterior segment at $1310 \mathrm{~nm}$. Arch Ophthalmol 2001;119:1179-85.

10 Bland JM, Altman DG. Statistical methods for assessing agreement between two methods of clinical measurement. Lancet 1986;1:307-10.

11 Shields CL, Shields JA, Materin M, et al. Iris melanoma: risk factors for metastasis in 169 consecutive patients. Ophthalmology 2001;108:172-8.

12 Marigo FA, Finger PT. Anterior segment tumors: current concepts and innovations. Surv Ophthalmol 2003;48:569-93.

13 Conway RM, Chew T, Golchet P, et al. Ultrasound biomicroscopy: role in diagnosis and management in 130 consecutive patients evaluated for anterior segment tumours. Br J Ophthalmol 2005;89:950-5.

14 Ursea R, Heinemann MH, Silverman $\mathrm{RH}$, et al. Ophthalmic, ultrasonographic findings in primary central nervous system lymphoma with ocular involvement. Retina 1997;17:118-23.

15 Lorente-Ramos RM, Armán JA, Muñoz-Hernández A, et al. Ultrasound of the eye made easy: a comprehensive how-to review with ophthalmoscopic correlation. Radiographics 2012;32:E175-200.

16 Kivelä T. Diagnosis of uveal melanoma. Dev Ophthalmol 2012;49:1-15.

17 Piñeiro-Ces A, Blanco-Teijeiro MJ, Mera-Yáñez MP, et al. [Ultrasound diagnosis in vasoproliferative tumours of the ocular fundus]. Arch Soc Esp Oftalmol 2011:86:247-53.

18 Sconfienza LM, Lacelli F, Ardemagni A, et al. High-resolution, three-dimensional, and contrast-enhanced ultrasonographic findings in diseases of the eye. J Ultrasound 2010;13:143-9.

19 Marigo FA, Finger PT, McCormick SA, et al. Iris and ciliary body melanomas: ultrasound biomicroscopy with histopathologic correlation. Arch Ophthalmol 2000;118:1515-21.

20 Razzaq L, Emmanouilidis-van der Spek K, Luyten GP, et al. Anterior segment imaging for iris melanocytic tumors. Eur J Ophthalmol 2011;21:608-14. 